\title{
BEAM TEST RESULTS FROM A PROTOTYPE FOR THE DELPHI MICROVERTEX DETECTOR
}

\author{
V. Chabaud, H. Dijkstra, M. Gröne, M. Flohr, R. Horisberger, L. Hubbeling, \\ G. Maehlum, A. Peisert, A. Sandvik and P. Weilhammer \\ CERN, CH-1211 Geneva 23, Switzerland
}

A. Czermak, P. Jalocha, P. Kapusta, M. Turala and A. Zalewska Institute of Nuclear Physics, Cracow, Poland

\author{
E. Sundell \\ Åbo Akademi, Turku, Finland \\ T. Tuuva \\ University of Helsinki, Finland
}

M. Battaglia, M. Caccia, W. Kucewicz, C. Meroni, N. Redaelli, R. Turchetta, A. Stocchi, C. Troncon and G. Vegni INFN, Milan, Italy

G. Baricello, M. Mazzucato, M. Pegoraro and F. Simonetto INFN, Padua, Italy

P. Allport and M. Tyndel

Rutherford Appleton Laboratory, Chilton, Didcot, Oxon., OX11 0QX, UK

H.J. Seebrunner

Fachhochschule Heilbronn, Heilbronn, FRG.

\begin{abstract}
Results are presented from a test in the CERN SPS North Area of a prototype of the DELPHI Microvertex Detector. Full-sized modules built up from prototype AC-coupled detectors and VLSI readout electronics were used. The spatial resolution of the detectors equipped with prototype VLSI chips was measured to be $6.5 \mu \mathrm{m}$. The system aspects, including the readout, were found to work well. Extrapolating to the final components we expect to achieve a measurement precision of $5 \mu \mathrm{m}$ with the DELPHI microvertex detector.
\end{abstract}




\section{INTRODUCTION}

The DELPHI microvertex detector will consist of two cylindrical shells of silicon microstrip detectors close to the beam pipe. Silicon detectors, with their very high spatial resolution, have been used successfully in the past in fixed target charm and beauty experiments [1], to extract cleanly the small fraction $(\leqslant 1 \%)$ of events containing charm or beauty. At LEP $42 \%$ of all hadronic events are expected to originate from $c \bar{c}$ or $b \bar{b}$ fragmentation and therefore contain at least 2 particles with lifetimes in the range $10^{-13}$ to $10^{-12} s$. The ability to tag primary quark flavours on an event by event basis and to study the spectroscopy of the heavy flavour hadrons will enhance the physics potential of the DELPHI detector [2].

The vertex detector is shown schematically in Fig. 1. Details can be found in Refs. 3 and 4 . It has been designed to measure the $R \phi$ coordinate of charged tracks with a precision of $5 \mu \mathrm{m}$, as close to the interaction point as possible. In practice this is limited by the relatively large diameter beam pipe required for the initial safe operation of the LEP machine. There will be 2 layers of microstrip detectors at radii of 9 and $11 \mathrm{~cm}$ with a length of $24 \mathrm{~cm}$. Each layer is built up of 24 overlapping modules. A module consists of 4 microstrip detectors with strips parallel to the beam axis. The strip pitch of $25 \mu \mathrm{m}$ was chosen to give the required spatial resolution of $5 \mu \mathrm{m}$. The basic detector unit, a half-module, consists of a pair of capacitively coupled silicon detectors [5] daisy-chained together. The readout pitch is $50 \mu \mathrm{m}$. Each readout strip is connected to a charge sensitive amplifier. These amplifiers have been built on a fully custom designed VLSI circuit, the MX3 [6]. Each chip consists of 128 amplifiers and multiplexing circuitry. The complete vertex detector consists of 192 individual silicon microstrip detectors with a total surface area of $0.33 \mathrm{~m}^{2}$ and 55,296 readout channels. These are multiplexed at the readout chip so that all the channels are read out on 48 twisted pair cables. The readout rate is $2.5 \mathrm{MHz}$. Data reduction is achieved online by zero suppression and cluster identification using a purpose-built programmable FASTBUS unit, the SIROCCO 4 [7].

The aim of the beam test was to measure the spatial resolution of the silicon detectors directly and to gain experience in the operation of such a large system. Ideally we would have liked to test all the final components, but many items were still under development. Including a high-precision 
reference beam telescope, a total of 10 silicon detectors was instrumented and read out (10,880 channels). Algorithms were developed off-line for estimating and continuously updating pedestals and rms noise. Cluster finding algorithms were also developed. A first attempt was made at understanding the problems associated with the alignment and stability of the vertex detector.

\section{BEAM TEST HARDWARE}

The experimental arrangement used during the beam test is shown schematically in Fig. 2. A minimal prototype of the DELPHI microvertex detector was prepared consisting of one inner and two outer modules. Each half-module consists of a pair of AC-coupled silicon detectors daisychained together giving $12 \mathrm{~cm}$ long readout strips. Only one of the 2 halfmodules in each module was equipped with electronics and read out. The detectors were produced by SI (Oslo) and have already been described elsewhere [5]. The mechanical assembly was the same as it will be for the complete detector when mounted in the DELPHI experiment. The modules were mounted on precision end-rings which in turn were supported by a replica of the inner wall of the Inner Detector.

The electronic readout chain was based on that proposed for the DELPHI experiment but several of the key items were only available in prototype form. The CMOS microplex chip used in the test, the MX2, has the same functionality as the final chip, the MX3 which was not yet available for the test beam experiment. The performance of both chips is summarised in Fig. 3. The MX3 has lower noise at the expense of a lower bandwidth. The power consumption, $0.5 \mathrm{~mW} / \mathrm{channel}$ and the radiation tolerance (greater than $55 \mathrm{Krad}$ ) are the same for both chips.

The readout processor foreseen is implemented in FASTBUS and has many functions. It accepts the string of multiplexed data into a 4-event deep front-end buffer. A signal processor then controls the data processing - typically the calculation and storage of pedestals and noise values, the subtraction of pedestals and the identification of clusters. The results are again stored in a 4-event deep output buffer. For this test a prototype CAMAC unit, without processor was used and all the raw data was recorded directly on magnetic tape so that algorithms for the data reduction could be developed off-line. 
The trajectory of the beam tracks was defined using crossed pairs of silicon detectors [9] as shown in Fig. 2. These had $25 \mu \mathrm{m}$ readout pitch and were equipped with NMOS microplex chips [8]. These reference detectors were read out through the same data acquisition system.

The two position monitoring systems foreseen for the vertex detector were installed and evaluated. A capacitive probe monitored movement of the vertex detector assembly with respect to the reference counters. An optical system composed of quartz fibres with a $50 \mu \mathrm{m}$ diameter core and $1.5 \mathrm{~mm}$ diameter focussing lenses and prisms, projects light spots onto the silicon detectors themselves. It was installed in such a way that it could monitor movement between the vertex detector and its support.

\section{ANALYSIS CHAIN}

For each trigger the raw pulse-height data from all the 10,880 channels were written to tape. Neither the pedestal subtraction nor the zero suppression features of the Sirocco were used. The off-line software therefore had the tasks of correctly associating each data element with a physical strip number; of calculating pedestals and noise values, subtracting pedestals and selecting valid signals; of finding clusters, calculating hit coordinates and fitting tracks.

The raw data in each channel consists of three contributions :

1) An overall $\mathrm{DC}$ level common to all 128 channels of a single microplex chip multiplexed onto one output. This varies from trigger to trigger and can be caused by external noise sources.

2) A pedestal or zero level, reflecting fluctuations in channel characteristics across the VLSI circuits. For the reference counters, which are not capacitively coupled, there is also a contribution from the leakage currents of each strip.

3) The charge collected as a result of the passage of a minimum ionizing particle through the detector (i.e. the signal).

The first component is calculated on a trigger by trigger basis by finding the average pulse-height per chip in ADC counts. The average is calculated for each chip using only "valid" channels (see below) and is then globally subtracted from all the data from that chip. The individual 
pedestals are calculated by averaging the residual pulse-height in each channel over many triggers. Updating of the average pedestal and of pedestal RMS is performed :

$$
\begin{gathered}
P e d^{N+1}=P e d^{N}+\frac{\left(\text { Pulse-height }-D C^{N+1}\right)-P e d^{N}}{W_{1}} \\
\left(\sigma^{N+1}\right)^{2}=\left(\sigma^{N}\right)^{2}\left(1-\frac{1}{W_{2}}\right)+\frac{\left(\left(\text { Pulse-height }-D C^{N+1}\right)-P e d^{N}\right)^{2}}{W_{2}}
\end{gathered}
$$

The choice of $\mathrm{W}_{1}$ and $\mathrm{W}_{2}$ depends on the level of fluctuations in the pedestal and noise values. Different values were tried. Setting both to 50 gives noise values which agree well with those found from calculating the standard deviation of the residual signal per channel taken over many triggers.

After these corrections the data is unpacked into the physical detector strip numbering and the reference counters are scanned for clusters. Strips which are dead, saturated or have noise greater than $10 \mathrm{ADC}$ counts (i.e. 3 times the average) counts are excluded as "not valid". Of the 8,960 reference detector channels, about 500 are affected. A cluster is then defined as the sum of all contiguous valid strips satisfying both of the following conditions :

1) Signal in each strip greater than $2.58 \times \sigma$ i.e. the probability of a noise fluctuation should be less than $1 \%$.

2) Signal in each strip greater than one eighth of the peak value of the minimum ionising pulse-height distribution for that detector. The mean value of this peak is 52 with a spread of $5.7 \mathrm{ADC}$ counts.

Only clusters, where the integrated signal exceeds half the peak value are kept. With this definition, the average number of strips per cluster is 2.4 \pm 0.2 . For estimating the hit position via charge division, only the neighbouring 2 strips with the largest signals are used. 
After preliminary alignment, the reference counter clusters are used to define tracks through the half-modules. These are then used to select the range of strips in the half-module through which the particle has passed.

\section{RESULTS WITH REFERENCE COUNTERS}

Performance of the reference counters was found to be well matched to our requirements. After cutting out bad channels an average noise for the reference counters of $3.3 \mathrm{ADC}$ counts was measured giving an averaged signal/noise ratio of 15.8 . Figure 4 shows the cluster pulse-height distribution for a reference counter.

After the cluster reconstruction a straight line fit is performed through the four planes of reference detectors measuring the $\mathrm{x}$-coordinate. Fitted tracks are accepted if : 1) their slope is less than $10 \mathrm{mrad}, 2$ ) at least three of the four planes have a reconstructed cluster, 3) the maximum residue between the extrapolated track position and the hit on each of the reference planes is less than 50 microns.

For the runs considered here, high energy ( $>60 \mathrm{GeV}$ ) beam particles were used so that multiple scattering effects were negligible. The correction for the true resolution of the reference counters from the measured residues is then straightforward, taking account of the least-squares fit procedure used which employs all counters to define the track parameters. In the absence of multiple scattering and assuming all detectors have the same intrinsic resolution :

$$
\begin{gathered}
\sigma_{x}^{i}=\frac{R M S(\text { Residue })}{\left(1-\frac{\sigma_{z}^{2}+\left(z_{i}-\bar{z}\right)^{2}}{N \sigma_{z}^{2}}\right)^{\frac{1}{2}}} \\
\sigma_{z}^{2}=\frac{\sum_{i=1}^{N}\left(z_{i}-\bar{z}\right)^{2}}{N}
\end{gathered}
$$


The correction factors that must be applied in our case to the residue distributions to get the true resolution estimates for each detector are 1.481 $(i=1,4)$ and $1.356(i=2,3)$. Note, as the counters were grouped in pairs, almost measuring at a single $z$ coordinate when compared with the distance between each pair the resolution is approximately $\sqrt{2} \times$ RMS of the residue distribution. The resolution of the 4 horizontal counters was found to be equal to better than $\sim 5 \%$.

The mechanical alignment of the reference counters was very good, and the detectors were found to be parallel to within $0.25 \mathrm{mrad}$. These small corrections were applied to the data. The fully corrected residue distribution for one of the reference detectors is shown in Fig. 5. It has a full width at half maximum of $9.2 \pm 0.3 \mu \mathrm{m}$. The expected relationship r.m.s. $=$ f.w.h.m. $/ 2 \sqrt{2 \ln 2}$ did not hold as there were non-Gaussian tails to the residue distribution. This is known to come from the dependence of the detector resolution on the track impact point with respect to the readout strips. This is particularly pronounced if one assumes that the division of the collected charge depends linearly on the relative strip distances. In fact it was found that the f.w.h.m. increased by $>50 \%$ between samples of tracks traversing midway between strips and those passing through a strip. Crude corrections were applied for the non-linearity in the relation between charge distribution and position which did reduce the nonGaussian tails of the residue distribution but did not alter the values of the f.w.h.m. From the FWHM we obtain a resolution of $3.9 \pm 0.1 \mu \mathrm{m}$,compared with the best value of $2.6 \mu \mathrm{m}$ [1] measured using DC-coupled detectors and discrete component electronics.

\section{RESUITS WITH HALF-MODULES}

The capacitively-coupled detectors for the DELPHI microvertex detector have been designed with very narrow $(\sim 6 \mu \mathrm{m})$ diodes to achieve low interstrip capacitance - on average $1.3 \mathrm{pF} / \mathrm{cm}$ has been measured. This has to be compared with 2.5 to $3 \mathrm{pF} / \mathrm{cm}$ measured on other detectors with similar layout [5]. From Fig. 3 it can be seen that for a minimum ionising signal of 24,000 electrons, the $\mathrm{S} / \mathrm{N}$ expected from the MX3 for a $12 \mathrm{~cm}$ long detector is $16: 1$. This has been confirmed directly by exposing a detector assembly to an Amercium source $(60 \mathrm{keV} \gamma)$. The signal to noise ratio measured with Am was 11.5:1 (Fig. 6), which extrapolates to a ratio of 16:1 
for minimum ionizing particles. The $\mathrm{S} / \mathrm{N}$ expected for the test beam assembly, using the then available MX2, is 8:1 (Fig. 3).

The measured average noise for these detectors was 4.0 $\pm 0.2 \mathrm{ADC}$ counts. The signal, as estimated from the peak of the pulse-height distribution (Fig. 7) obtained in the experiment was $28 \pm 2 \mathrm{ADC}$ counts giving a signal/noise of about $7: 1$, in good agreement with the measurements using external capacitors.

The spatial resolution achieved in a large system depends on many factors apart from the electronic noise. It is therefore important to independently measure the spatial resolution directly. Also, since no attempt was made to align the half-modules with respect to the reference system, our data provided a useful opportunity to explore alignment techniques. We found that the strips were not parallel to the reference y direction and corrections of up to $2 \mathrm{mrad}$ were needed. Also we saw evidence of movement of all three half-modules between different runs taken at different times. Given the mounting of the half-shells within a long tube supported on a metallic moveable stage, this is not surprising. Temperature variations between day and night alone gave rise to relative motions of half-shell and reference system of $\sim 10 \mu \mathrm{m}$. This was measured by the in situ capacitive probe monitoring system. Possible movements of the halfshell with respect to its support tube were investigated using the light spot monitoring system. No displacements were found.

Applying all the cuts and corrections described above (in particular, an event by event correction of displacements observed with capacitive probes), and cleaning the data further by requiring a $\mathrm{S} / \mathrm{N} \geq 5$ for single strip clusters to be accepted, the residue distribution shown in Fig. 8 is found for the DELPHI module \#4. Calculating the spatial resolution from the FWHM of this distribution we find an RMS of $6.5 \mu \mathrm{m}$. A Gaussian fit to this distribution yields $\sigma=8.3 \pm 0.4 \mu \mathrm{m}$, which leads to a resolution of $\sigma=8$ $\mu \mathrm{m}$ after subtraction of the error of the predicted impact point. The fraction of events in the tails of the experimental distribution outside the Gaussian fit is $9 \%$. We performed a cross-check on our result by fitting the residues of events whose impact in the DELPHI module \#4 is within \pm 10 $\mu \mathrm{m}$ of the centre of a readout strip (sample A) and events with an impact of $\pm 15 \mu \mathrm{m}$ around the middle line between readout strips (sample $\mathrm{B}$ ). We find from Gaussian fits $\sigma=10.5 \pm 1 \mu \mathrm{m}$ for sample A and $6.8 \pm 0.6 \mu \mathrm{m}$ for sample 
B. In Fig. 9 the calculated resolution for $\mathrm{Si}$ strip detectors as a function of $\mathrm{S} / \mathrm{N}$ is shown for $50 \mu \mathrm{m}$ pitch readout and $25 \mu \mathrm{m}$ pitch readout. Both our results for the reference detectors and the DELPHI module agree well with the calculated resolution. From Fig. 9 and Fig. 4 we conclude that the DELPHI modules equipped with the MX3 CMOS VLSI will achieve the desired resolution of $\sigma=5 \mu \mathrm{m}$.

\section{CONCLUSIONS}

Prototypes of many of the components to be used in the DELPHI Microvertex Detector project have been built and tested together under realistic experimental conditions. The system worked well and we were able to derive the signal/noise and spatial resolution of both NMOS microplex equipped $25 \mu \mathrm{m}$ direct-coupled detectors and CMOS (MX2) microplex equipped $50 \mu \mathrm{m}$ daisy-chained capacitively-coupled detectors with one intermediate diode. The resolution values extracted from FWHM were $3.9 \mu \mathrm{m}$ and $6.5 \mu \mathrm{m}$. The latter resolution is limited by the electronics noise of the prototype MX2 chip. The improved signal/noise performance for the final DELPHI modules equipped with the MX3 VLSI circuit, measured to be $16: 1$, implies that the detector modules will give the $5 \mu \mathrm{m}$ resolution specified in the DELPHI Microvertex Detector proposal [3].

\section{Acknowledgements}

We wish to acknowledge the valuable assistance we received from many people. The test would not have succeeded without the good beam provided by the SPS (our especial thanks to N. Doble) and the tolerance of our DELPHI colleagues sharing the beam line. We would like to thank J. Lidbury and N. Beadle at RAL and K. Ratz at CERN for the design and construction of the precision mechanics. We are indebted to P. Seller and J. Stanton for the microplex chips MX2 and MX3. R. Boulter provided us with beautifully mounted detector assemblies. Of course we would have had no data without the help of M. Jonker from the DELPHI on-line group. We also wish to thank A. Bjorkebo for the tape management and finally C. Ponting for her hard work on this paper and the figures. 


\section{REFERENCES}

[1] P. Weilhammer, Experience with Si Detectors in NA32, Workshop on New Solid-State Devices for HEP, Berkeley, California, USA, 1985, p. 83;

R. Alberganti et al., Nucl. Instrum. Methods A248 (1986) 337;

E691 Contribution to the XXIIIrd Int. Conf. on HEP, Berkeley (1986).

[2] H. Dijkstra et al., Physics Aspects of the DELPHI Microvertex Detector, Nucl. Instrum. Methods A277 (1989) 160.

[3] DELPHI Microvertex Detector, Addendum to the Technical Proposal, DELPHI 86-86 GEN-2 (Oct. 1986).

[4] M. Burns et al., Progress in the Construction of the DELPHI Microvertex Detector, Nucl. Instrum. Methods A277 (1989) 154.

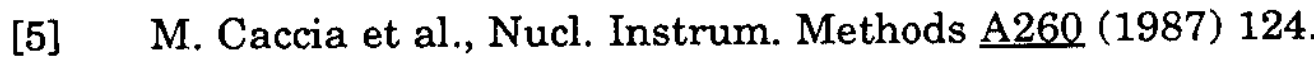

[6] J. Stanton, A Low Power Low Noise Amplifier for a 128 Channel Read-Out Integrated Circuit, RAL-89-009 (1989).

[7] N. Bingefors and M. Burns, Sirocco $N$, Hardware and Software Manual, DELPHI 88-48 TRACK-48 (1988).

[8] J.T. Walker et al., Nucl. Instrum. Methods $\underline{26}$ (1984) 200.

[9] These detectors were produced by MICRON SEMICONDUCTOR. 


\section{Fiqure Captions}

Fig. 1 : Schematic view of a half-shell of the DELPHI microvertex detector.

Fig. 2 : The detector arrangement for the beam test (not to scale).

Fig. 3 : MX2 performance vs capacitance (MX3 from Ref. 6).

Fig. 4 : Pulse height distribution for reference detectors (all clusters > $\Sigma$ min.I.) obtained in beam test.

Fig. 5 : Residues for a reference detector.

Fig. 6. : Americium pulse height distribution measured in DELPHI equipped with MX3.

Fig. 7 : Pulse height distribution for half-modules (only clusters on tracks) from beam particles.

Fig. 8 : Residues for the DELPHI half-module.

Fig. 9 : Calculated resolution for $25 \mu \mathrm{m}$ diode pitch silicon strip detectors. 


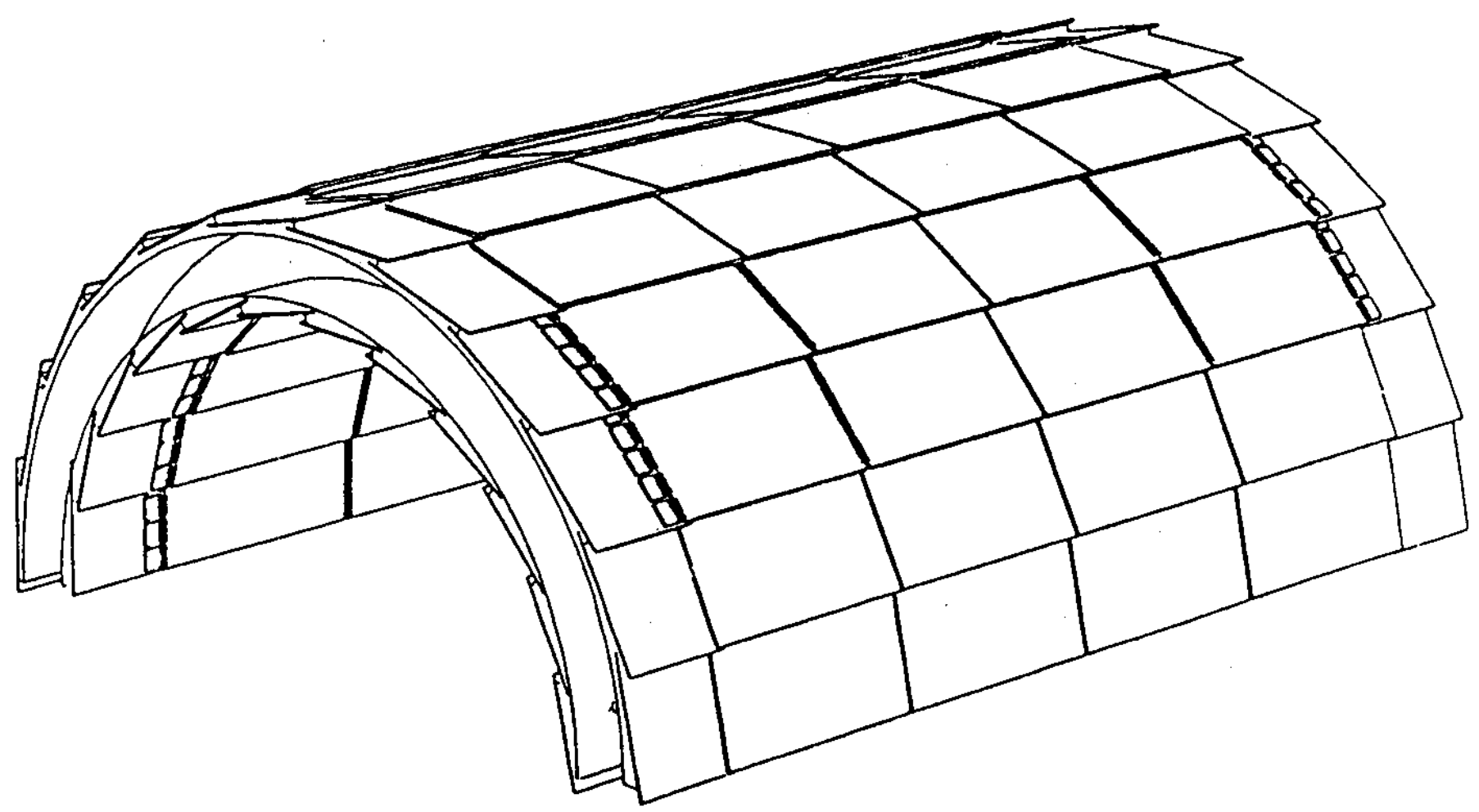

Fig. 1 


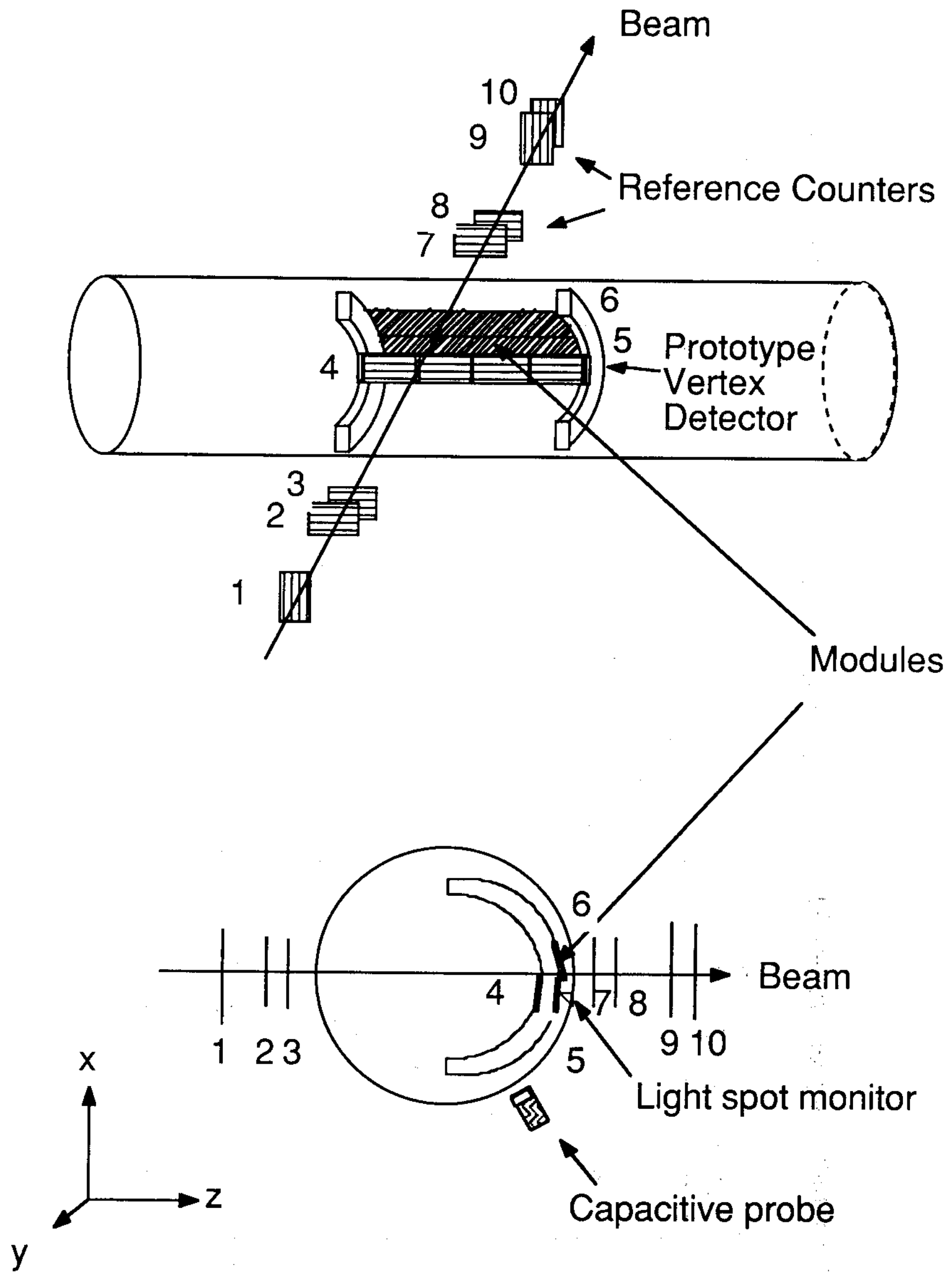

Fig. 2 

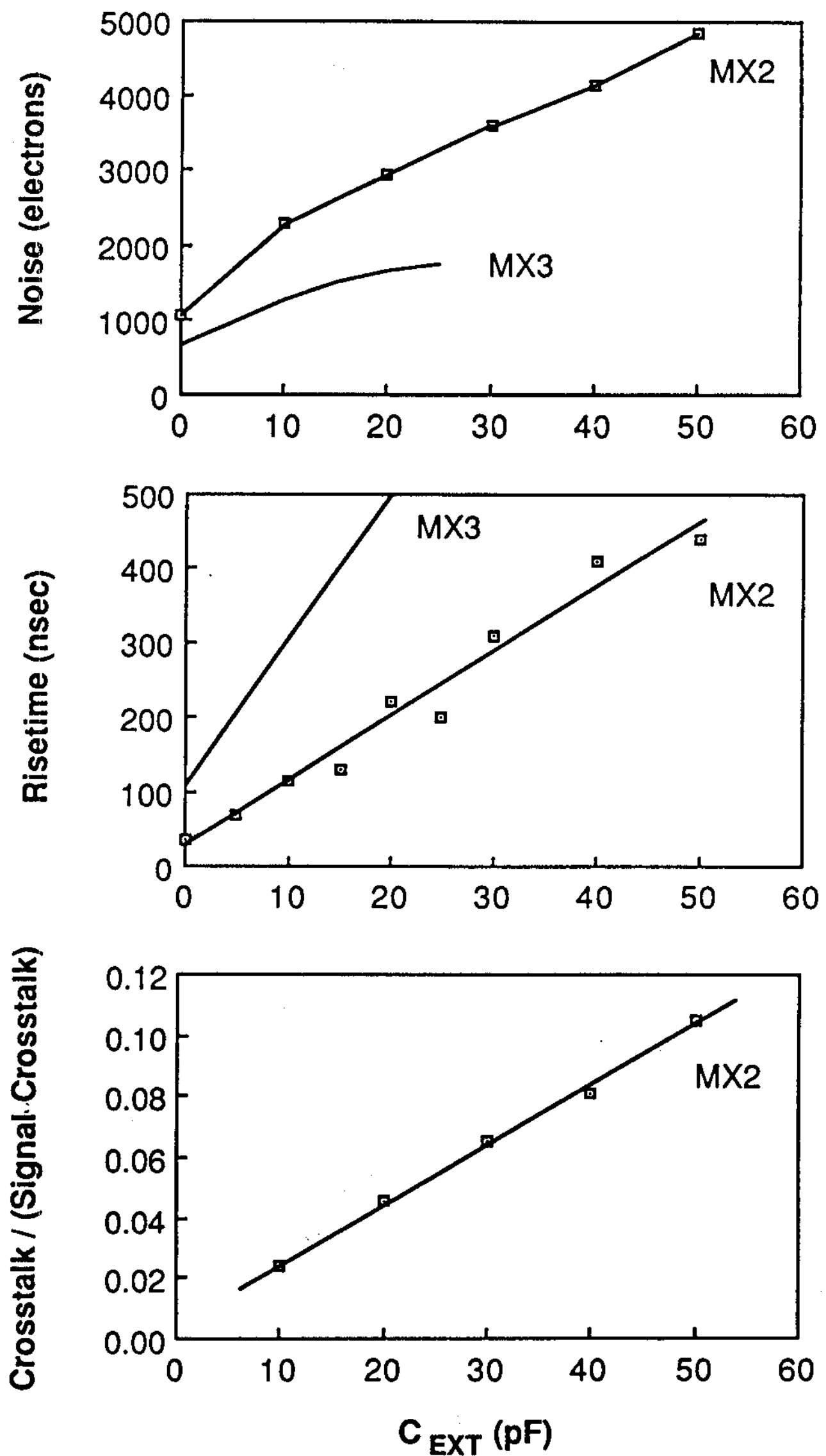

Fig. 3 


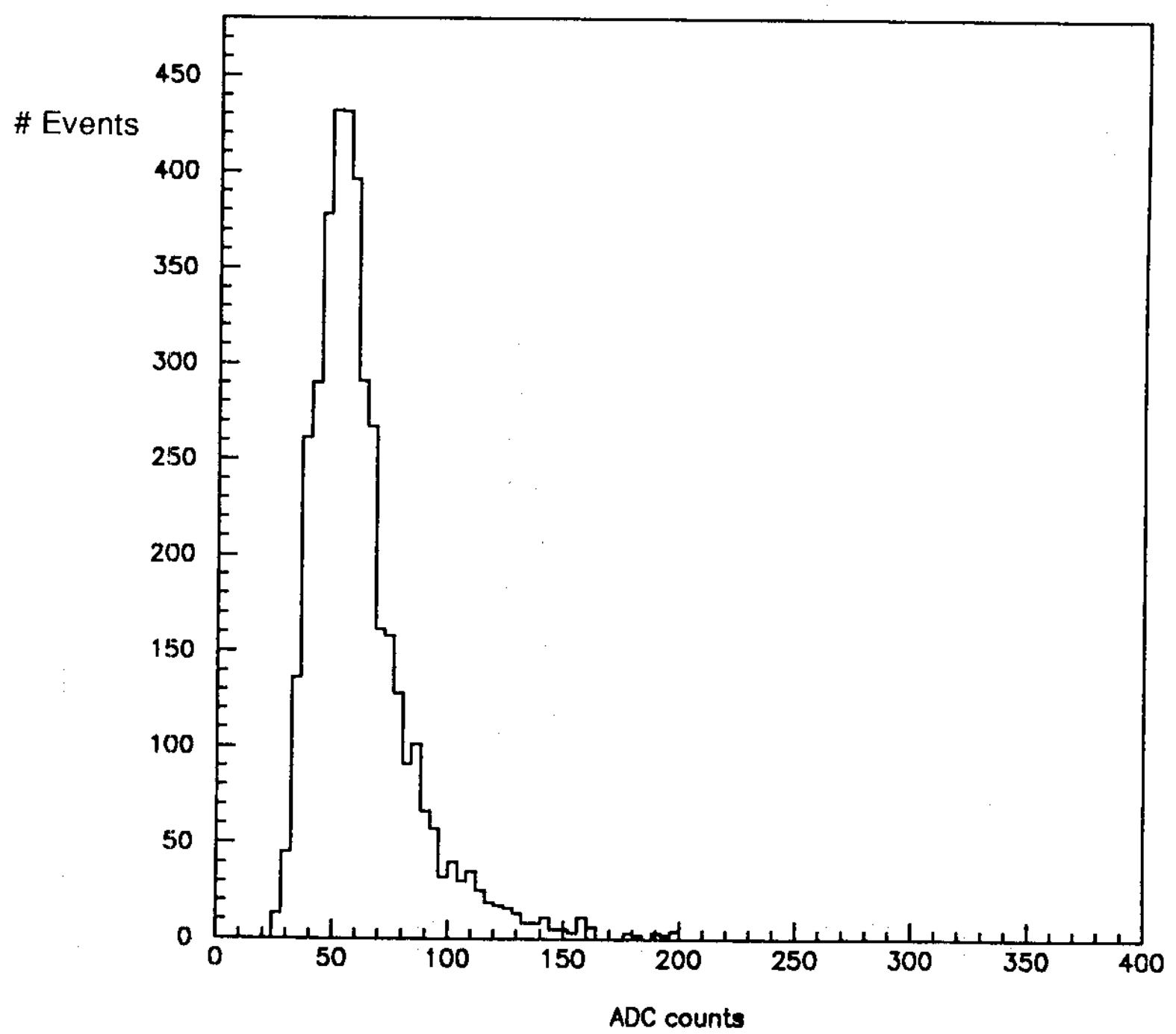

Fig. 4 


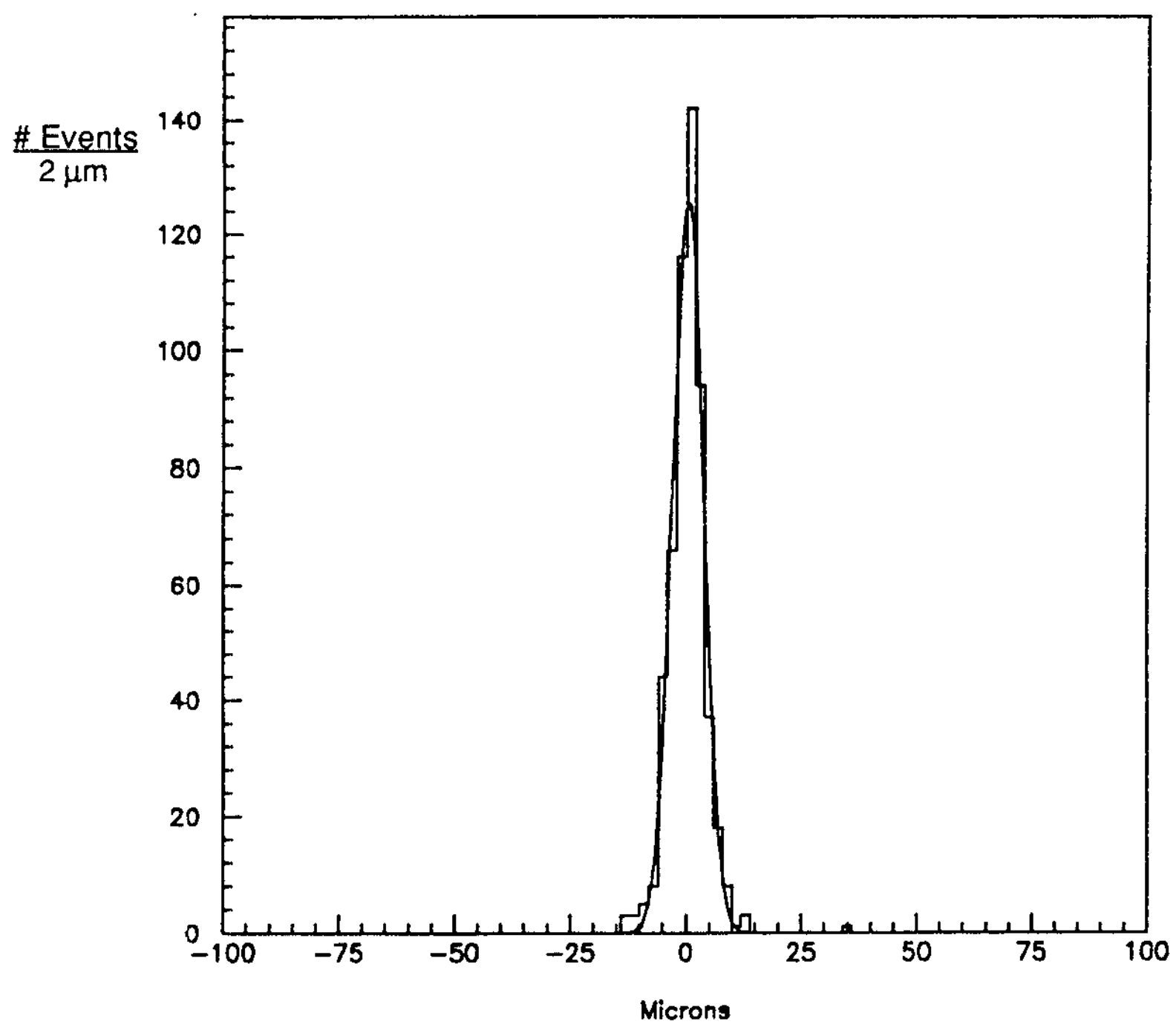

Fig. 5 


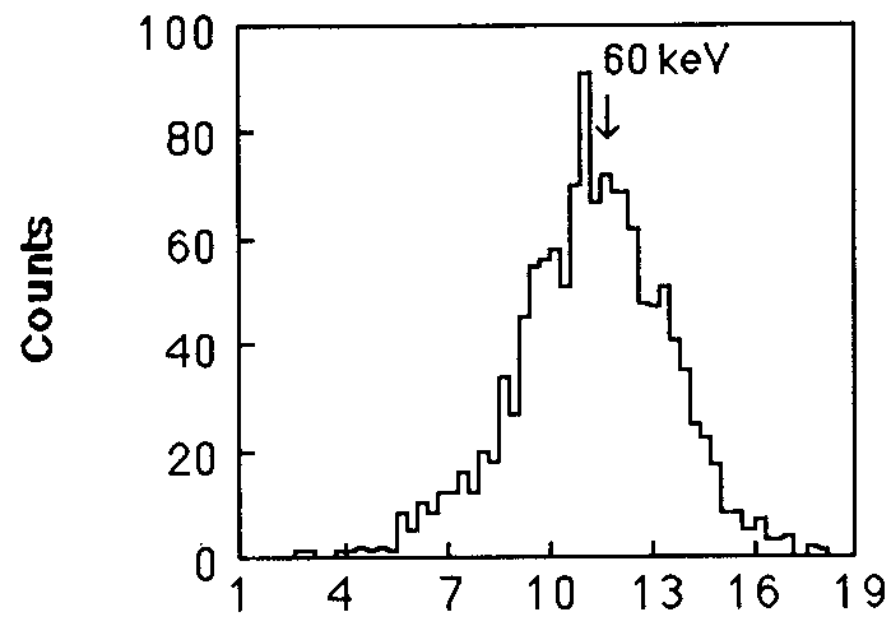

RMS Noise

Fig. 6 


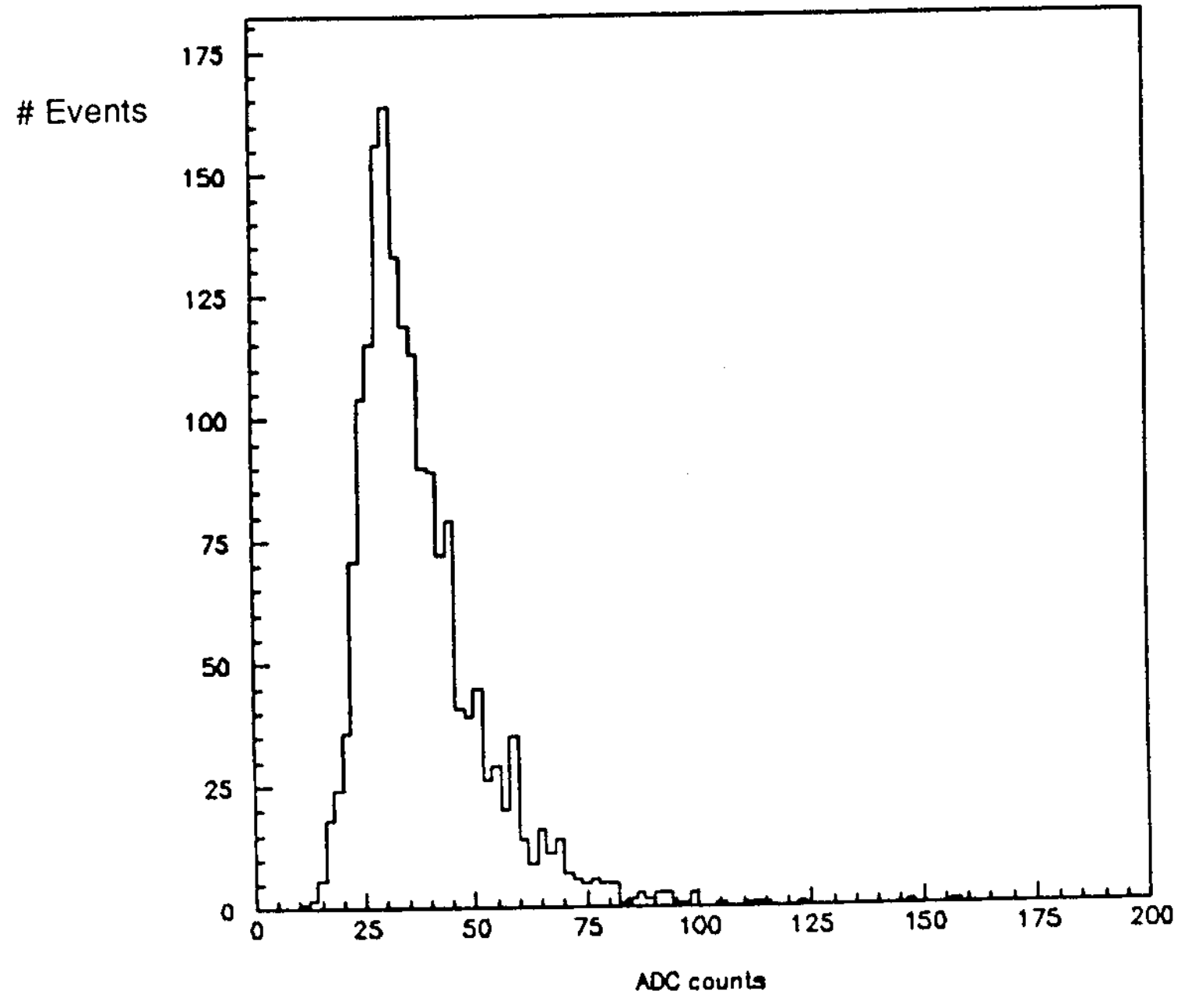

Fig. 7 


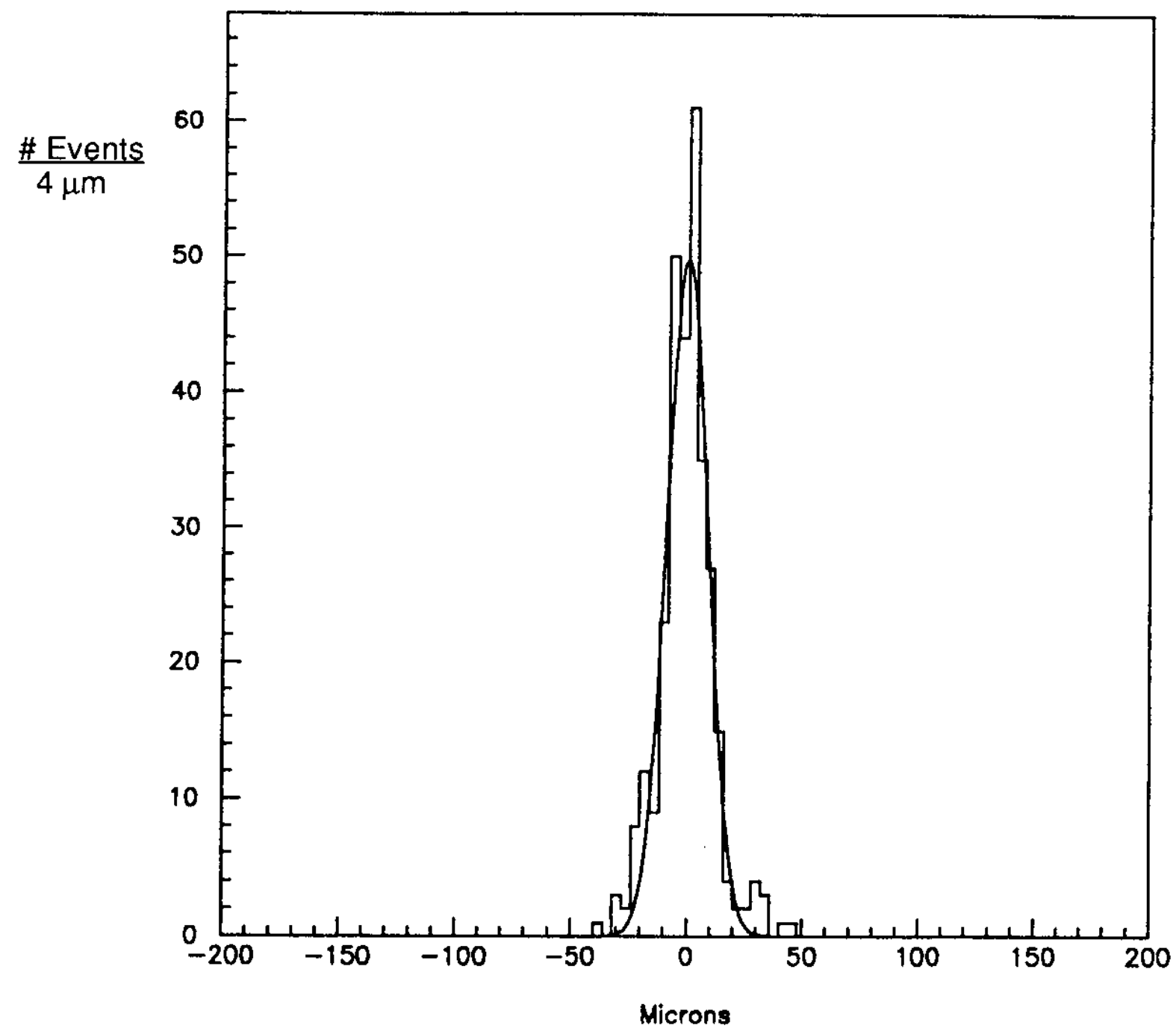

Fig. 8 


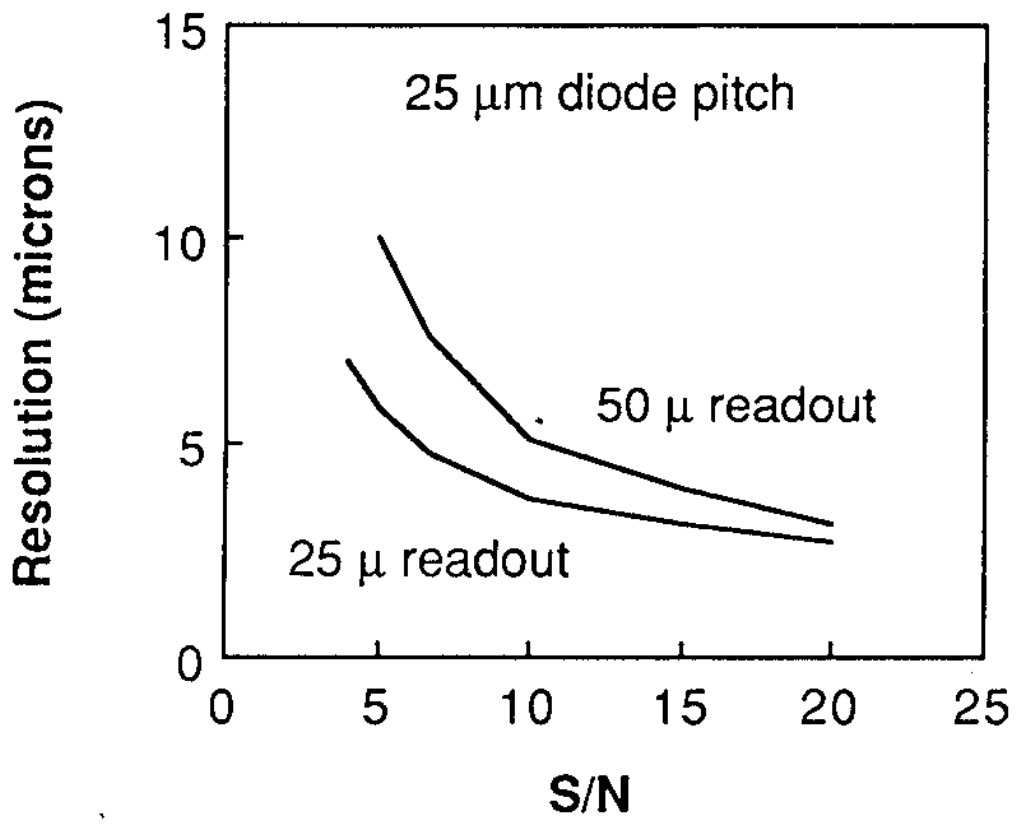

Fig. 9 